\title{
Coexistence of Electron-Glass Phase and Persistent Photoconductivity in GeSbTe Compounds
}

\author{
Z. Ovadyahu \\ Racah Institute of Physics, The Hebrew University, Jerusalem 91904, Israel
}

\begin{abstract}
It is demonstrated that persistent-photoconductivity (PPC), well-studied in lightly-doped semiconductors, is observable in GeSbTe compounds using infrared excitation at cryogenic temperatures. The low level of energy-flux necessary to induce an appreciable effect seems surprising given the high carrier-concentration $\mathrm{n}$ of these ternary alloys $\left(\mathrm{n}>10^{20} \mathrm{~cm}^{-3}\right)$. On the other hand, their high density of carriers makes GeSbTe films favorable candidates for exhibiting intrinsic electron-glass effects with long relaxation times. These are indeed observed in GeSbTe thin-films that are Anderson-localized. In particular, a memory-dip is observed in samples with sheet resistances larger than $\approx 10^{5} \Omega$ at $\mathrm{T} \approx 4 \mathrm{~K}$ with similar characteristics as in other systems that exhibit intrinsic electron-glass effects. Persistent-photoconductivity however is observable in GeSbTe films even for sheet resistances of the order of $10^{3} \Omega$, well below the range of disorder required for observing electron-glass effects. These two non-equilibrium phenomena, PPC and electron-glass, are shown to be of different nature in terms of other aspects as well. In particular, their relaxation dynamics is qualitatively different; the excess conductance $\Delta \mathrm{G}$ associated with PPC decays with time as a stretched exponential whereas a logarithmic relaxation law characterizes $\Delta \mathrm{G}(\mathrm{t})$ of all electron-glasses studied to date. Surprisingly, the magnitude of the memory-dip is enhanced when the system is in the PPC state. This counter-intuitive result may be related to the compositional disorder in these materials extending over mesoscopic scales. Evidence in support of this scenario is presented and discussed.
\end{abstract}

PACS numbers: 72.20.-i 72.40.+w 78.47.da 72.80.Ng

\section{INTRODUCTION}

Many phenomena in solid state systems exhibit monotonous conductance changes, suggestive of a nonequilibrium phenomenon. Annealing of defects during heat-treatment, irradiation by an external source, diffusion of injected particles, and illumination by light, are familiar examples of such phenomena.

There are two specific phenomena that exhibit sluggish conductance relaxation following excitation by a light-source: persistent-photoconductivity (PPC) that has been studied extensively as of 1968 [1-13], and the less familiar (but just as controversial) electron-glass 14 23]. In addition to the similar way these effects are reflected in the sample conductance once triggered by light, both become more prominent at lower temperatures and both exhibit a non-exponential relaxation. This sometimes led to assuming a common mechanism to these phenomena 24].

In this work we describe some light-induced nonequilibrium transport properties in thin films of $\mathrm{GeSb}_{\mathrm{x}} \mathrm{Te}_{\mathrm{y}}$. The study was initiated as a test of the conjecture that intrinsic electron-glass effects, with long relaxation-times, are generic and should be observable in all Anderson insulating systems with sufficiently large carrier-concentrations [25, 26]. The ternary compound $\mathrm{GeSb}_{\mathrm{x}} \mathrm{Te}_{\mathrm{y}}$ reported by several groups to have carrierconcentration $\mathrm{n}$ in the range $\approx 10^{20}-10^{21} \mathrm{~cm}^{-3}[27$ [29] appeared to be a suitable candidate for this test. This expectation turned out to be correct; in their insulating state, $\mathrm{GeSb}_{\mathrm{x}} \mathrm{Te}_{\mathrm{y}}$ films do exhibit electron-glass behavior. However, a surprisingly large photosensitivity was encountered in the very first experimental run. The sample was initially tested by a short exposure to infrared radiation using a power level that in other electronglasses yielded a few percent increase in conductance $\mathrm{G}$. In the $\mathrm{GeSb}_{\mathrm{x}} \mathrm{Te}_{\mathrm{y}}$ sample this resulted in the conductancereading promptly going off-scale. It soon became clear that this photo-response is a manifestation of persistentphotoconductivity rather than the anticipated electronglass phenomenon. Persistent-photoconductivity is referred to in this work for processes that involve charge generation by exposure to light without change of stoichiometry; this should not be confused with instances where self-doping is affected when e.g., oxygen is expelled from the sample by exposure to high-energy photons as happens in $\mathrm{In}_{2} \mathrm{O}_{3-\mathrm{x}}$ [30] and $\mathrm{ZnO}$ [31]. The latter processes may appear to be similar in that they show sluggish conductance relaxations, but they are of a different nature.

Persistent-photoconductivity typically occurs in systems with carrier-concentration smaller than $\approx 10^{19} \mathrm{~cm}^{-3}$ like lightly-doped semiconductors [1 13]. Intrinsic electron-glass effects were never observed in these lowdensity systems [26].

Effects associated with the electron-glass phase were duly observed in field-effect experiments on Andersonlocalized $\mathrm{GeSb}_{\mathrm{x}} \mathrm{Te}_{\mathrm{y}}$ samples. These revealed a memorydip with the same characteristic features found in previously studied electron-glasses. $\mathrm{GeSb}_{\mathrm{x}} \mathrm{Te}_{\mathrm{y}}$ is the first narrow-gap semiconductor that exhibits intrinsic electron-glass behavior. By "intrinsic" we mean that the glassy effects appear in a given substance independently of the way the sample was prepared to achieve the required parameters (resistance at the measuring tempera- 
ture, carrier-concentration, and dimensionality). Most importantly, the system has to exhibit a memory-dip with a width that is commensurate with the carrierconcentration of the material [26] .

That electron-glass phase and persistentphotoconductivity coexist in the $\mathrm{GeSb}_{\mathrm{x}} \mathrm{Te}_{\mathrm{y}}$ compounds gives us a unique tool to learn about both phenomena. The current study demonstrates that PPC and electron-glass effects are different phenomena. Using the empirically known behavior of the electron-glass and persistent-photoconductivity it is argued that some of the non-trivial transport effects observed when the two coexist may be a result of the inhomogeneous nature of the system.

\section{EXPERIMENTAL}

\section{Sample preparation and characterization}

Samples used for this study were prepared by e-gun depositing a $\mathrm{GeSb}_{2} \mathrm{Te}_{5}$ alloy unto room temperature substrates in a high-vacuum system (base pressure 0.8$1 \cdot 10^{-7} \mathrm{mbar}$ ) with rates of $2-3 \AA /$ second. A constant thickness of $120 \AA$ was used for all films in this study. Two types of substrates were used; $1 \mathrm{~mm}$-thick microscope glass-slides, and $0.5 \mu \mathrm{m} \mathrm{SiO}_{2}$ layer thermally grown on $<100>$ silicon wafers. The Si wafers were boron-doped with bulk resistivity $\rho \simeq 2 \cdot 10^{-3} \Omega \mathrm{cm}$, deep into the degenerate regime. These wafers were employed as the gate electrode in the field-effect measurements. The fieldeffect technique was used heavily in this study as an analytical tool. The microscope glass-slides were mostly used for optical characterization and for Hall-Effect measurements, both performed at room-temperatures.

Each deposition batch included samples for optical excitation measurements, samples for Hall-effect measurements, and samples for structural and chemical analysis. For the latter study, carbon-coated $\mathrm{Cu}$ grids were put close to the sample during its deposition. The TEM grids received the same post-treatment as the samples used for transport measurements.

The source material was $99.999 \%$ pure $\mathrm{GeSb}_{2} \mathrm{Te}_{5}$ (American Elements). Different preparation runs however, produced films that usually had different stoichiometry than the source material. In the samples used in this work the GeTe atomic ratio, for example, varied between 1:3.5 to 1:4.8. These films should therefore be referred to as $\mathrm{GeSb}_{\mathrm{x}} \mathrm{Te}_{\mathrm{y}}$. By itself, variations in film composition seem to make little difference to the transport properties; we were not able to identify a qualitative influence of the film global stoichiometry on the transport results reported below, the main effect on the latter turns out to be the film sheet-resistance $\mathrm{R}_{\square}$.

Transmission-electron-microscopy (TEM), using the Philips Tecnai F20 G2) were employed to characterize

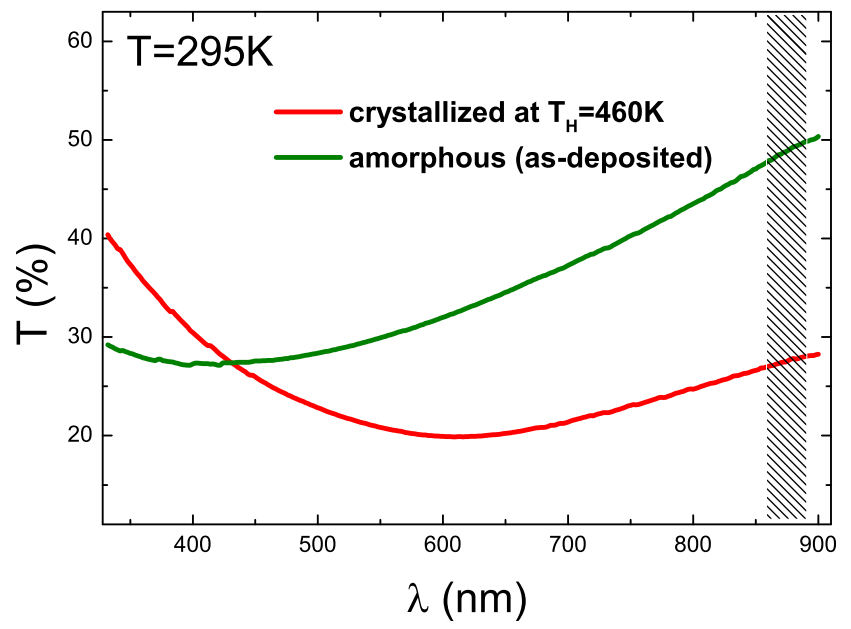

FIG. 1: The optical transmission in the visible (relative to the bare glass-slide) of the as-prepared amorphous and the crystallized film (at $\mathrm{T}_{\mathrm{H}}=455 \mathrm{~K}$ ). The hatched area indicates the spectral range of the IR LED used in this work. The absorption of the $\mathrm{GeSb}_{\mathrm{x}} \mathrm{Te}_{\mathrm{y}}$ film at these wavelengths is smaller than $10 \%$. The difference in the transmission between the two films is due to stronger reflection, absorption, and scattering of the crystalline material.

the films composition and microstructure. The Varian Cary-1 spectrophotometer was used for optical measurements.

Polycrystalline samples of $\mathrm{GeSb}_{\mathrm{x}} \mathrm{Te}_{\mathrm{y}}$ were obtained by subjecting the as-deposited (amorphous) films to heattreatment at a temperature $\mathrm{T}_{\mathrm{H}}$ for 2-3 minutes during which the sample was crystallized. The difference in the optical properties in the visible due to the amorphouscrystalline transformation could be seen in films deposited on glass substrates as a mild change in the color tint. An example is shown in Fig.1.

The heat-treatment temperature was held constant during the heat-treatment process to $\pm 2 \mathrm{~K}$ around a specific value $\mathrm{T}_{\mathrm{H}}$. This was limited to the range $430-490 \mathrm{~K}$ for the samples used in this work. In agreement with reports by other groups [28, 32], the resistivity of a given crystalline film was found to depend on the value of $\mathrm{T}_{\mathrm{H}}$. Using a heating-stage attachment in the TEM revealed that onset of crystallization could be as low as 400-410K, and that the grain size increases monotonically with $\mathrm{T}_{\mathrm{H}}$.

A TEM micrograph and associated diffraction pattern of a typical film used in this study is given in Fig.2. This illustrates the polycrystalline nature of the film and a tight, space-filling packing of the crystallites with rare occasional cracks between adjacent crystallites. Grain-sizes as large as $\approx 10 \mathrm{~nm}$ start appearing at $\mathrm{T}_{\mathrm{H}} \approx 440 \mathrm{~K}$, and grain-sizes as big $\approx 1$ micron were observed for $\mathrm{T}_{\mathrm{H}} \approx 540 \mathrm{~K}$. Interestingly, at this grain-size, which is much larger than the film thickness $(\mathrm{d}=120 \AA)$, the film still maintains its mosaic, physically continuous structure 


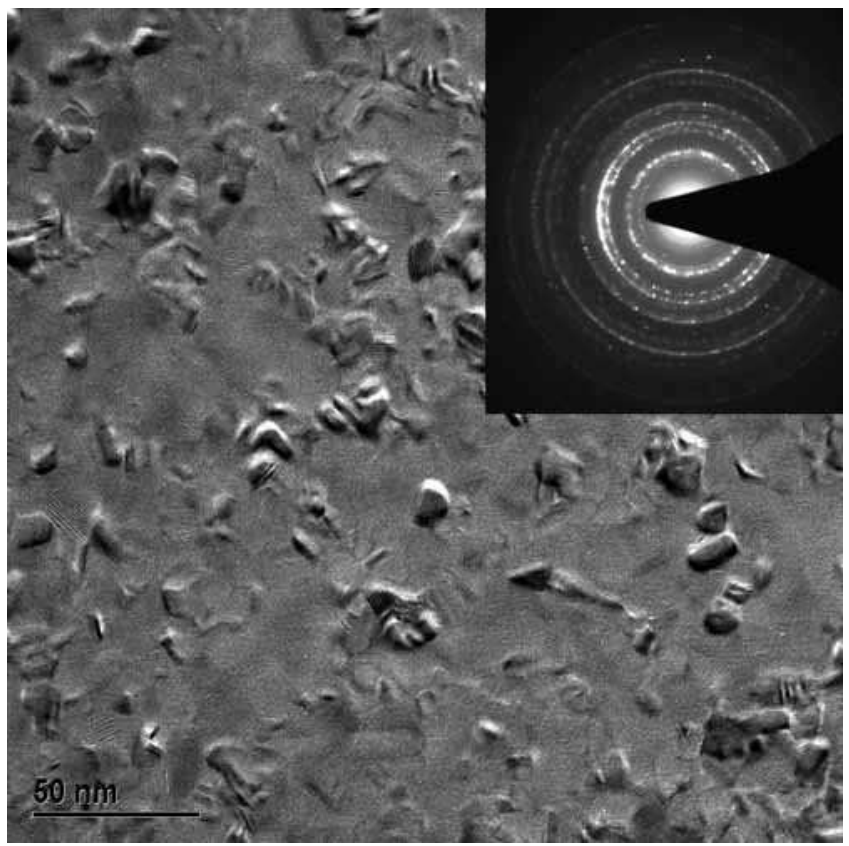

FIG. 2: Bright-field image and the associated diffraction pattern of a typical $\mathrm{GeSb}_{\mathrm{x}} \mathrm{Te}_{\mathrm{y}}$ film crystallized at $\mathrm{T}_{\mathrm{H}}=455 \mathrm{~K}$. The diffraction in this case was taken at 10 degrees inclination to expose the film texture.

The diffraction pattern taken from these films were consistent with the rhombohedral (R-3m) phase of GeTe in all our samples. No diffraction ring was found that could not be related to this basic structure. Note however that the presence of some amorphous component is always a possibility which is hard to rule out in these cases (especially when the film is supported on amorphous carbon). A small amount of texture (preferred orientation) was observed in these films (see diffraction pattern in Fig.2). There are quite a number of defects that are observed in the micrograph; grain boundaries and twinning being the most prevalent. These defects, as well as the compositional-disorder, discussed later, and surface scattering are presumably responsible for the low mobility of the films (a desirable feature in the context of this work).

Gold strips were deposited on the ion-bombarded glass substrates prior to the $\mathrm{GeSb}_{\mathrm{x}} \mathrm{Te}_{\mathrm{y}}$ deposition. Lateral dimensions of the samples used here were $0.5-1 \mathrm{~mm}$ long and $1 \mathrm{~mm}$ wide. Samples had sheet resistance $\mathrm{R}_{\square}$ in the range $2 \mathrm{k} \Omega-25 \mathrm{M} \Omega$ at $4 \mathrm{~K}$. This range includes diffusive samples $\left(\mathrm{R}_{\square}<\mathrm{h} / \mathrm{e}^{2}\right)$ as well as samples well into the hopping regime $\left(\mathrm{R}_{\square} \gg \mathrm{h} / \mathrm{e}^{2}\right)$ at the measurement temperature. The room-temperature carrier-concentration $\mathrm{n}$ of these films varied in the range of $(4-9) \cdot 10^{20} \mathrm{~cm}^{-3}$, a range too narrow to show a definite correlation with the sample resistance.

\section{Measurement techniques}

Conductivity of the samples was measured using a two terminal ac technique employing a 1211-ITHACO current preamplifier and a PAR-124A lock-in amplifier. All measurements were performed with the samples immersed in liquid helium at $\mathrm{T} \approx 4.1 \mathrm{~K}$ held by a 100 liters storagedewar. This allowed up to two months measurements on a given sample while keeping it cold (and in the dark) which was utilized to extend the time-duration of relaxation processes.

The ac voltage bias in conductivity measurements was small enough to ensure near-ohmic conditions (except for the current-voltage plots). Optical excitations in this work were accomplished by exposing the sample to an AlGaAs diode operating at $\approx 0.88 \pm 0.05 \mu \mathrm{m}$ (see Fig.1), mounted on the sample-stage typically $\approx 10-15 \mathrm{~mm}$ from the sample. The diode was energized by a computercontrolled Keithley 220 current-source.

\section{RESULTS AND DISCUSSION}

\section{Non-equilibrium induced by optical-excitation in $\mathrm{GeSb}_{\mathbf{x}} \mathrm{Te}_{\mathbf{y}}$}

The experimental protocol used for optical excitation is illustrated in Fig. 3 using a $120 \AA$ film of $\mathrm{GeSb}_{\mathrm{x}} \mathrm{Te}_{\mathrm{y}}$, deep in the insulating regime. The experiment begins $\approx 24$ hours after the sample is cooled-down to $4.1 \mathrm{~K}$ by recording $\mathrm{G}(\mathrm{t})$ for 1-2 minutes to establish a baseline, near-equilibrium conductance $\mathrm{G}_{0}$. The IR source was then turned on for 3 seconds, then turned off while $G(t)$ continues to be measured. The brief IR burst causes $G$ to promptly increase by $\delta \mathrm{G}_{\mathrm{IR}}$ which decays slowly with time once the source is turned off (Fig.3).

This change in the sample conductance has a rather small effect on the conductance versus voltage characteristics as shown in Fig.4. The deviations from Ohm's law at $4.1 \mathrm{~K}$ start to become substantial at a field of $\approx 500 \mathrm{~V} / \mathrm{m}$, which is similar to what is observed in other disordered films of comparable resistance at these temperatures 26]. However, these deviations remain rather small at higher fields possibly suggesting a relatively small hopping length.

On first sight, the behavior of G(t) depicted in Fig.3 appears to be similar to that of the "IR-protocol" applied to an electron-glass sample (see, for example, Fig.1 of [33]). A closer look reveals some differences. First, the effect is much larger than what is usually encountered with any of the previously studied electron-glass; note that $\mathrm{a} \approx 3 \mathrm{~mJ}$ of infrared flux was sufficient to obtain $\delta \mathrm{G}_{\mathrm{IR}} / \mathrm{G}_{0} \approx 80 \%$ (Fig.3). For comparison, an energyflux of $\approx 80 \mathrm{~mJ}$ achieved only $\delta \mathrm{G}_{\mathrm{IR}} / \mathrm{G}_{0} \simeq 15 \%$ in the most IR-responsive electron-glass studied to date (see, Fig.6 of [33]). 


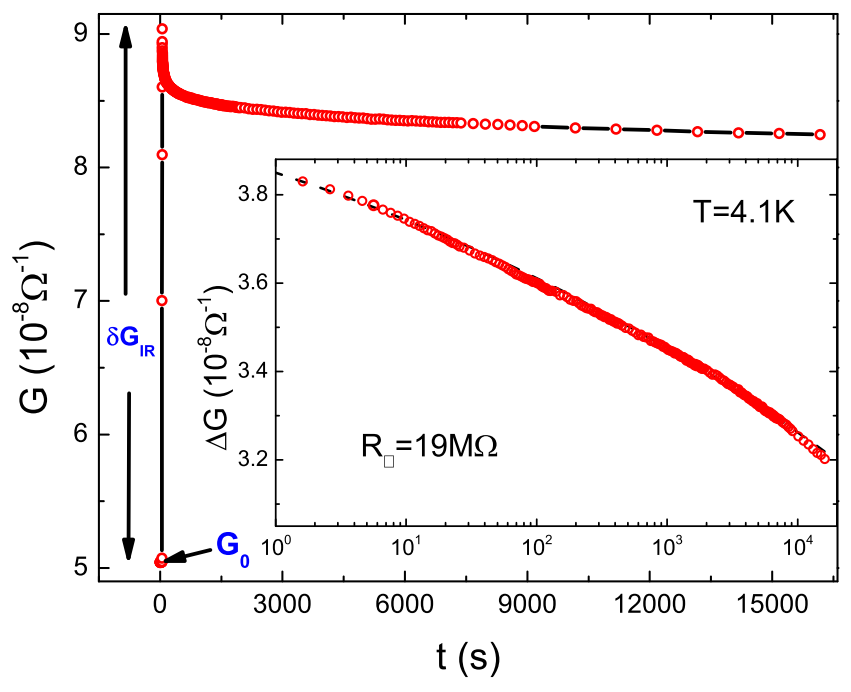

FIG. 3: The IR-protocol used on a $19 \mathrm{M} \Omega$ sample to illustrate the response of the conductance and its relaxation. The IR source is turned on for 3 seconds at an intensity of $1 \mathrm{mWatt}$ then turned off. The inset shows the relaxation of the excess conductance as function of time illustrating that the experimental curve systematically deviates from the logarithmic dependence (the origin of the time axis in this plot is taken as 1sec after the IR source is turned off). The dashed line is a mathematical fit to $\delta \mathrm{G}(\mathrm{t}) \propto \exp \left[-(\mathrm{t} / \tau)^{\beta}\right]$ with $\beta=0.1$ and $\tau=4 \cdot 10^{9}$ sec.

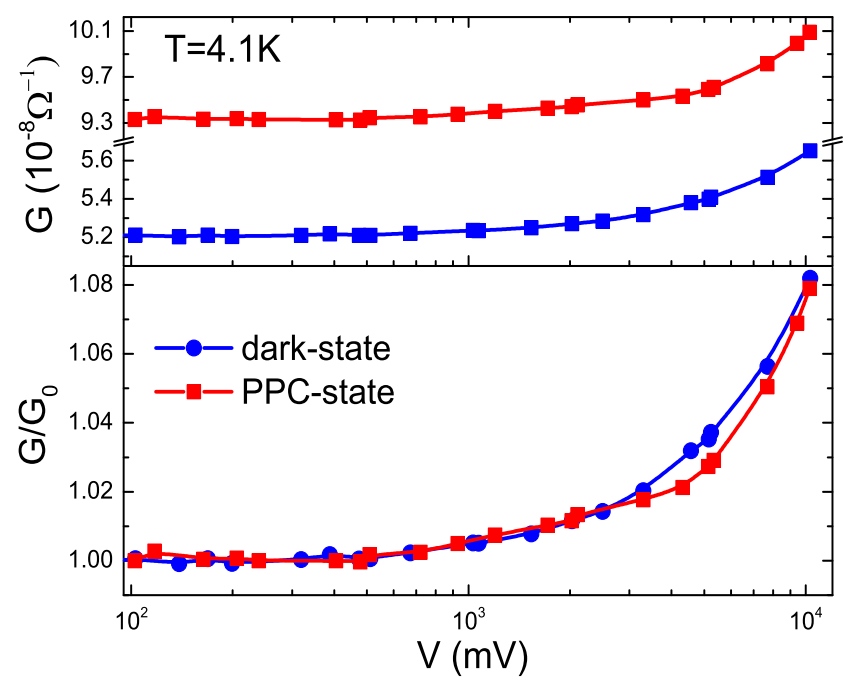

FIG. 4: The dependence of the conductance of a GeSb $\mathrm{Te}_{\mathrm{y}}$ film (the same sample as in Fig.3) on the applied voltage at the dark-state and several hours after IR exposure.

Secondly, the initial fast drop of the excess conductance (once the IR source is off), common to all IRexcitations involving electron-glasses [33], is not observed here. More importantly, the temporal relaxation-law of $\mathrm{G}(\mathrm{t})$ is different: The inset to Fig.3 demonstrates that the slow component of the induced excess conductance relaxes with time as a stretched exponent, $\mathrm{G}(\mathrm{t}) \propto \exp [-$ $\left.(\mathrm{t} / \tau)^{\beta}\right]$. This should be contrasted with the logarithmic law for $\mathrm{G}(\mathrm{t})$ that is a characteristic feature of the intrinsic electron-glass [26, 34]. The stretched exponential relaxation law has been consistently observed in more than twenty $\mathrm{GeSb}_{\mathrm{x}} \mathrm{Te}_{\mathrm{y}}$ samples measured in this study. These included samples with sheet resistance $R_{\square}$ covering the range $2.5 \mathrm{k} \Omega$ to $24 \mathrm{M} \Omega$ at $\mathrm{T} \approx 4 \mathrm{~K}$ all showing this relaxation law with the same $\beta=0.1 \pm 0.005$ as a bestfit parameter. Similar values for $\beta$ are typically found at these temperatures in the relaxation law of the PPC in $\mathrm{AlGa}_{\mathrm{x}} \mathrm{As}_{1-\mathrm{x}}$ compounds [5]. The other fit parameter in the stretched-exponential $\tau$, turned out to be in the range of $10^{8} \mathrm{sec}$ to $10^{10} \mathrm{sec}$, and showed no systematic correlation with $\mathrm{R}_{\square}$. Apparently the disorder (assessed by the value of the sample $R_{\square}$ ) affects this phenomenon mainly through the relative magnitude of the excess conductance.

Whether the stretched exponential law is an inherent property of this phenomenon or is just a concise way to parametrize the data is open to debate (it may be possible to fit $\delta \mathrm{G}(\mathrm{t})$ by 2-3 exponential terms [11]). What is clear however is that the functional form of the relaxation deviates appreciably from the well-established logarithmic relaxation law of intrinsic electron-glasses [26]. This is obvious in the extended measurements that were taken here, but might be missed in runs that are shorter than two-decades in time (due to the rather small value of $\beta$ ).

It is noteworthy that this nonequilibrium effect persists throughout a wide range of disorder, including both the insulating and the diffusive regime. This is illustrated in Fig.5 for a series of $\mathrm{GeSb}_{\mathrm{x}} \mathrm{Te}_{\mathrm{y}}$ films with different degrees of disorder but measured under identical IR-protocol conditions. This observation, by itself, is a strong evidence that the $\delta \mathrm{G}_{\mathrm{IR}}$ is not an electron-glass effect; the latter is strictly a hopping-regime phenomenon and vanishes as the diffusive regime is approached [26, 35, 36].

Actually, the effects described in Fig.3 and Fig.5 are manifestations of a well-known phenomenon - persistentphotoconductivity (PPC). This phenomenon has been studied for more than four decades, mostly in lightlydoped semiconductors. In the majority of cases, the relaxation law was found to be stretched-exponential [2, 4 13. as in this work. Different models were offered to account for PPC but they all share a common ingredient; a barrier that hinders the recombination of the photocreated electron-hole pairs. In essence, the excess conductance due to this mechanism is an enhanced carrierconcentration (in the hopping system $\delta \mathrm{G}_{\mathrm{IR}}$ may depend exponentially on $\delta \mathrm{n}$ ). The excess conductance in the electron-glass on the other hand is essentially due to enhanced mobility; the $\mathrm{G}(\mathrm{t})$ relaxation reflecting dressing of particles to form quasi-particles [37].

It is rather surprising to find $\mathrm{PPC}$ in degenerate semiconductors such as these $\mathrm{GeSb}_{\mathrm{x}} \mathrm{Te}_{\mathrm{y}}$ compounds with their high carrier-concentration. To the best of our knowledge, this is the first occurrence of PPC in a 


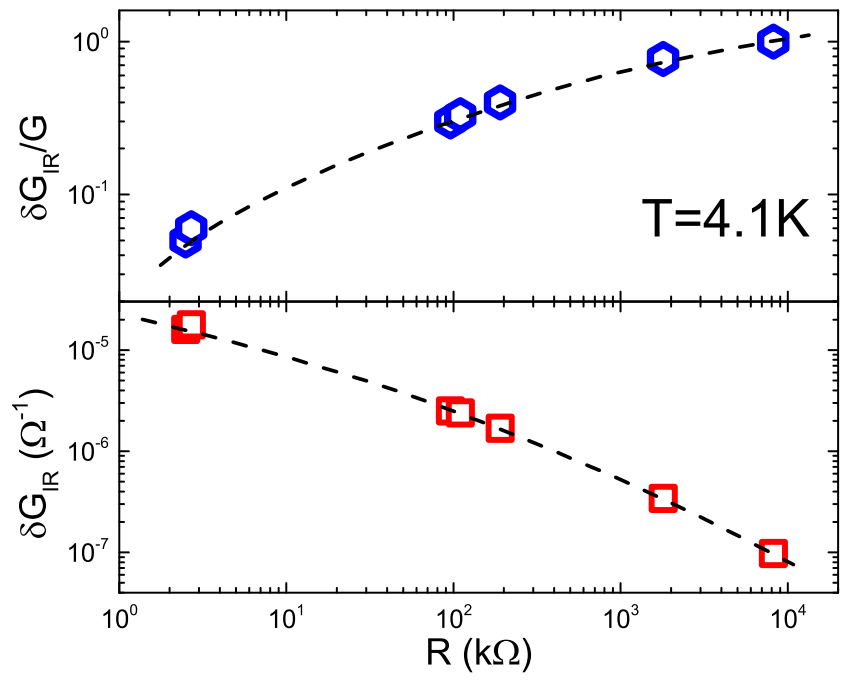

FIG. 5: The dependence of the PPC magnitude on film disorder (represented by $R_{\square}$ ). These are based just on samples where care was taken to use identical illumination conditions. See Fig. 3 for the definition for $\delta \mathrm{G}_{\mathrm{IR}}$ and $\mathrm{G}_{0}$.

system with carrier-concentration $\mathrm{n}>10^{20} \mathrm{~cm}^{-3}$. Typical values for $\mathrm{n}$ in systems exhibiting $\mathrm{PPC}$ range from $10^{15} \mathrm{~cm}^{-3}$ to $10^{19} \mathrm{~cm}^{-3}[3,7]$. These relatively low carrier-concentrations made it feasible to demonstrate, via Hall effect measurements, that the effect is associated with increase of carrier-concentration [3, 7]. High carrierconcentration, on the other hand, makes the $\mathrm{GeSb}_{\mathrm{x}} \mathrm{Te}_{\mathrm{y}}$ a prime candidate to exhibit intrinsic electron-glass effects, which was the original reason for choosing this system for the study.

In the following we present further experimental results demonstrating that electron-glass effects and PPC are distinct phenomena. They may be separated by controlling the conditions required for the appearance of each in turn. They may also coexist, making it possible to learn something on the material from their interplay.

$\mathrm{GeSb}_{\mathrm{x}} \mathrm{Te}_{\mathrm{y}}$ is an intrinsic Electron-Glass (when Anderson localized)

It is straightforward to eliminate the PPC - just not turn it on! This should leave the scene to the electronglass, which may be probed while in the "dark-state" by non-optical means. A dark-state of a pre-exposed sample may be restored by raising it above the helium bath and holding it for few minutes at $\mathrm{T}>30 \mathrm{~K}$ while in the shielded Dewar. $\mathrm{T} \approx 30 \mathrm{~K}$ is presumably of the order of the energy-barrier associated with the PPC in this system. Thermal recycling (from $4 \mathrm{~K}$ to $30-40 \mathrm{~K}$ ) was used to reinstate the sample in its dark-state if it was in the PPC state. This restored the original conductance and the field-effect characteristics the sample had prior to IRexposure to a very high accuracy, which suggests that

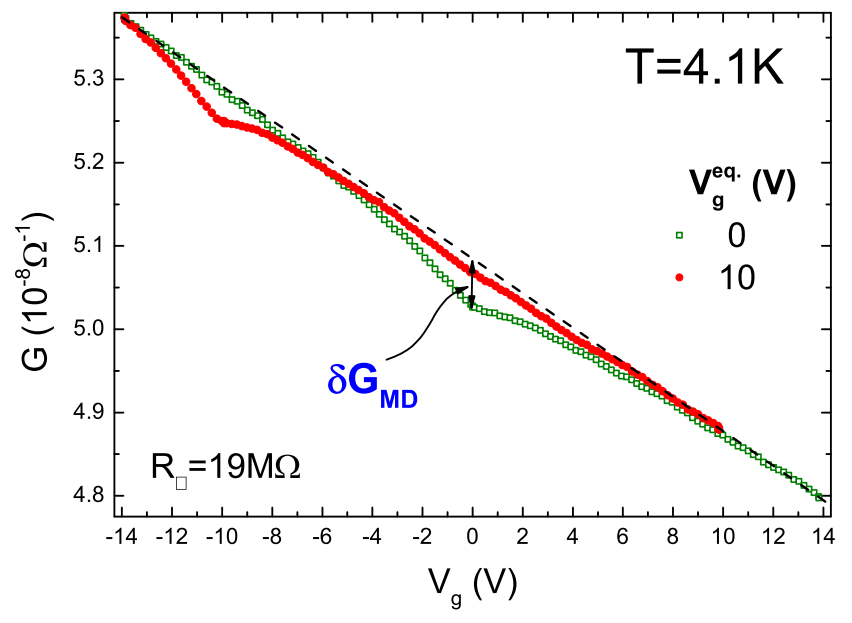

FIG. 6: Conductance as function of the gate voltage for the same sample as in Fig.3 (but here in its dark-state). The history of this protocol described in the text. The resulting field effect curves $G\left(V_{g}\right)$ demonstrate the characteristic feature of intrinsic electron-glass; a memory dip that appears as a local minimum around the gate-voltage $\mathrm{V}_{\mathrm{g}}^{\mathrm{eq}}$ under which the sample was allowed to equilibrate. The dashed line is the thermodynamic component of the field-effect.

there are no irreversible material changes in the sample due to the IR illumination.

The acid test for intrinsic electron-glass is the existence of a memory-dip [26]. This involves a field-effect measurement; recording the conductance as function of a gate-voltage $\mathrm{V}_{\mathrm{g}}$. Results of such measurements are shown below (Fig.6) for the same sample that was used in Fig.3.

Two traces are shown in Fig.6; the first was taken 20 hours after the sample was cooled down and allowed to equilibrate while $\mathrm{V}_{\mathrm{g}}=0 \mathrm{~V}$ was held between the sample and the gate. The resulting field-effect trace $\mathrm{G}\left(\mathrm{V}_{\mathrm{g}}\right)$ is composed of two components; an antisymmetric component reflecting the underlying (thermodynamic) density-of-states (DOS), and a superimposed dip, centered around the $\mathrm{V}_{\mathrm{g}}$ where the systems was allowed to relax $\left(\mathrm{V}_{\mathrm{g}}=0 \mathrm{~V}\right.$ in this particular case). The latter, the so called memory-dip, is the distinguishing feature of the intrinsic electron-glass [26]. The width of the memory-dip is comparable with that of the amorphous version of indium-oxide with a comparable carrierconcentration [38]. The second trace shown in Fig.6 was taken after the gate-voltage was moved to $\mathrm{V}_{\mathrm{g}}=-$ $10 \mathrm{~V}$ and left there overnight. A new $\mathrm{G}\left(\mathrm{V}_{\mathrm{g}}\right)$ trace was taken, 21 hours after letting the sample equilibrate under $\mathrm{V}_{\mathrm{g}}=-10 \mathrm{~V}$, to produce the curve labeled by full circles. This field-effect shows the same thermodynamic component as the old one while the memory-dip is now centered around the newly imposed gate-voltage. This illustrates the "two-dip-experiment" another common feature of electron-glasses [26]. 


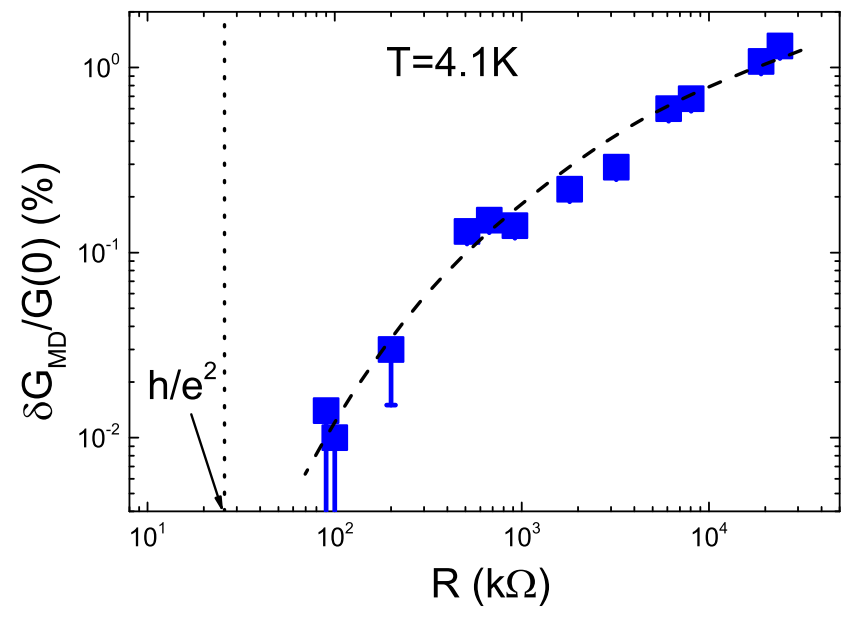

FIG. 7: The dependence of the memory-dip magnitude $\delta \mathrm{G}_{\mathrm{MD}} / \mathrm{G}_{0}$ on the film sheet-resistance $\mathrm{R}_{\square}$. These were obtained from $G\left(V_{g}\right)$ curves of the different samples taken with the same sweep rate $d V_{g} / d t$. The dashed curve through the data point is guide to the eye. The dotted separates the diffusive regime from the strongly localized one. A remarkably similar dependence on disorder was observed in other electron-glasses [34, 35].

Note that $\partial \mathrm{G} / \partial \mathrm{V}_{\mathrm{g}}$ of the thermodynamic component has the opposite sign to that observed in n-type semiconductors such as indium-oxide and thallium-oxide films [26]. This is consistent with the p-type nature of $\mathrm{GeSb}_{\mathrm{x}} \mathrm{Te}_{\mathrm{y}}$, where the Fermi energy lies at the valenceband [39].

A characteristic feature observed in all intrinsic electron-glasses is a monotonous dependence of the memory-dip (MD) magnitude on disorder (at fixed sweep-rate and temperature) and, in particular, that it vanishes at the diffusive regime [26, 35, 36]. The data in Fig.7 confirms that the same trend, and the same functional dependence for $\delta \mathrm{G}_{\mathrm{MD}} / \mathrm{G}(0)$ vs. $\mathrm{R}_{\square}$, is observed in $\mathrm{GeSb}_{\mathrm{x}} \mathrm{Te}_{\mathrm{y}}$ films.

In contrast with the electron-glass phase that categorically requires $R_{\square}>h / e^{2}$ for its existence, persistentphotoconductivity is observable in the entire disorder regime covered in this work which includes samples in the weakly-localized regime. The PPC relative magnitude $\delta \mathrm{G}_{\mathrm{PPC}} / \mathrm{G}_{0}$ does depend on the film disorder as shown in Fig.5 above but it is clearly observed in samples with $\mathrm{R}_{\square}<\mathrm{h} / \mathrm{e}^{2}$. In terms of the absolute value of the excess conductance it is actually larger in diffusive samples than in the strongly-localized regime. Once $\mathrm{R}_{\square}$ becomes smaller than $\approx 10^{5} \Omega$ the magnitude of the MD quickly approaches the limit of the experimental noise-level (Fig.7) while the excess-conductance associated with the PPC in these $\mathrm{GeSb}_{\mathrm{x}} \mathrm{Te}_{\mathrm{y}}$ films is not affected by the impending diffusive phase; neither the magnitude nor the parameters of the relaxation law seem to show any qualitative change (Fig.5).

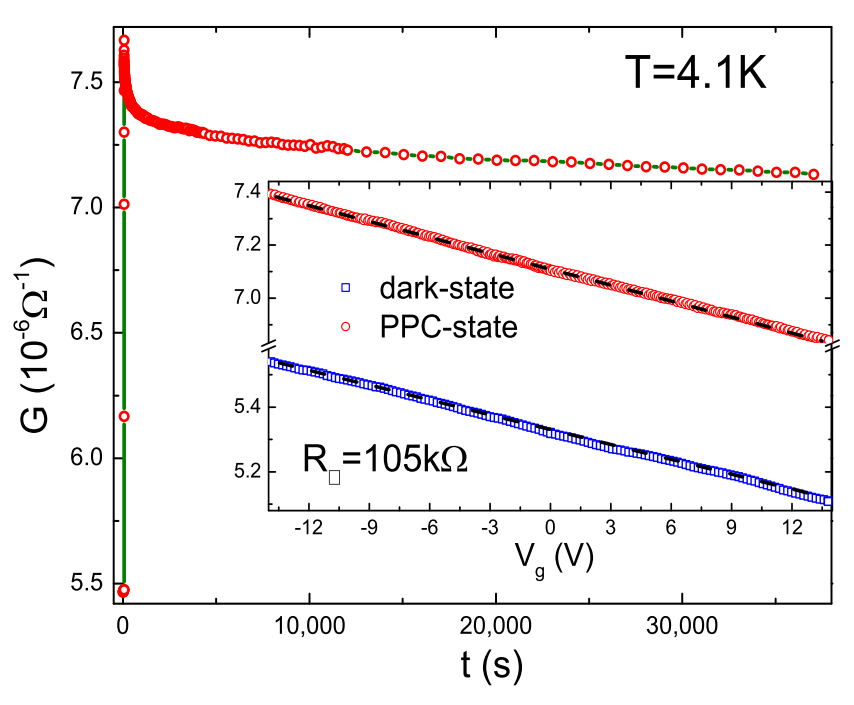

FIG. 8: Comparing the result of the IR-protocol (main figure) with the field-effect (inset) of a sample with an intermediate degree of disorder $\left(\mathrm{R}_{\square}=105 \mathrm{k} \Omega\right)$ before and after IRillumination. The relaxation of the conductance after the IR illumination is turned off is fitted by the same stretched exponential dependence as the $19 \mathrm{M} \Omega$ sample in Fig. 3 with $\beta=0.1$ and $\tau=1 \cdot 10^{9} \mathrm{sec}$. Note that the field-effect shows mainly a straight line with no clear signature of a memory-dip at $\mathrm{V}_{\mathrm{g}}=0 \mathrm{~V}$ where the sample was equilibrated.

This observation can be illustrated by using a sample in this intermediate disorder regime. Such data are shown in Fig.8. The data in this figure demonstrate that an intermediate disorder regime may be reached in a sample that still exhibits prominent PPC but the signature for electron-glass is not to be seen above the noise level.

Obviously, strong-localization, a mandatory requirement for showing electron-glass effects, is not a condition for observing persistent-photoconductivity in $\mathrm{GeSb}_{\mathrm{x}} \mathrm{Te}_{\mathrm{y}}$. Empirically, these two phenomena tend to favor vastly different carrier-concentration for their observation; intrinsic electron-glass behavior, with relaxation times larger than seconds, has been observed only in systems with high carrier-concentrations [26]. Persistentphotoconductivity on the other hand, has been reported almost exclusively in lightly-doped semiconductors. Observation of the latter phenomenon requires in addition, the existence of a specific defect-type to form a barrier that hinders fast recombination [8, 13].

These different preferences however, are apparently not mutually exclusive. As shown next, the two phenomena may coexist.

\section{Lighting-up the Memory-Dip}

The existence of a memory-dip in $\mathrm{GeSb}_{\mathrm{x}} \mathrm{Te}_{\mathrm{y}}$ with $\mathrm{R}_{\square}>10^{5} \Omega$ was anticipated; these samples are Andersonlocalized, and they have relatively high carrier- 


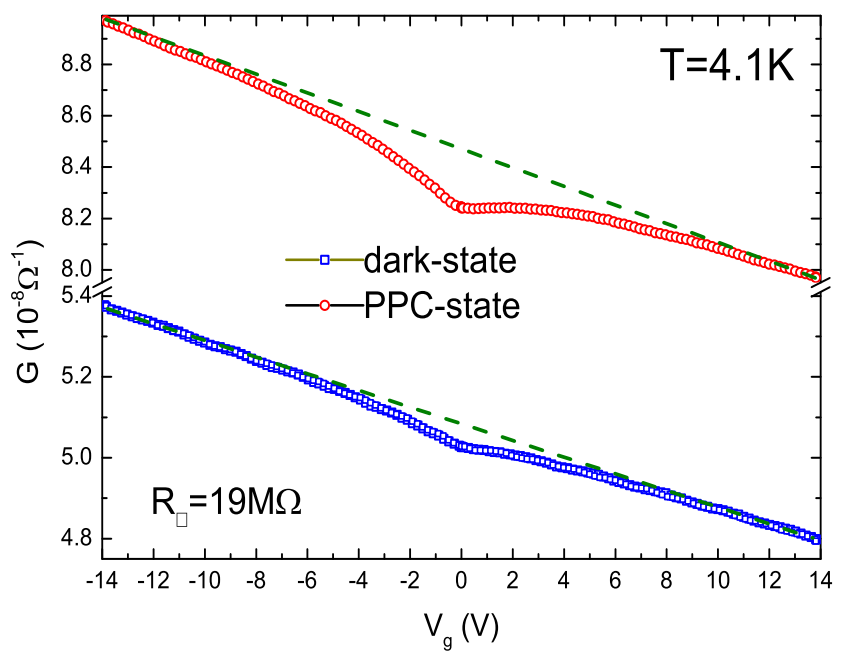

FIG. 9: Comparing the field-effect characteristics $\mathrm{G}_{\mathrm{DS}}\left(\mathrm{V}_{\mathrm{g}}\right)$ for the $R_{\square}=19 \mathrm{M} \Omega$ sample in the dark-state with $\mathrm{G}_{\mathrm{IR}}\left(\mathrm{V}_{\mathrm{g}}\right)$ taken one hour after exposing the sample to $1 \mathrm{mWatt}$ IR radiation for $3 \sec (\mathrm{a} \approx 1$ hour delay is necessary for obtaining meaningful $\mathrm{G}\left(\mathrm{V}_{\mathrm{g}}\right)$ data; immediately after terminating the illumination $G$ is still varying significantly over the $\approx 1$ minute required for obtaining a useful $G\left(V_{g}\right)$ reading across this range of $\left.V_{g}\right)$. The figure clearly demonstrates that a more developed memorydip is developed in the irradiated sample. Dashed lines are the thermodynamic component $\mathrm{G}\left(\mathrm{V}_{\mathrm{g}}\right)$ of the field-effect.

concentration. These are the empirically established conditions for observing the electron-glass phenomenon over conveniently long time-scales [26]. At this time there is no known exception to this observation.

Exposing these samples to infrared radiation however, had an unexpected effect. Figure 9 compares the fieldeffect measured on the sample that was used in Fig.6 in the dark-state with a $G\left(V_{g}\right)$ taken with the same experimental protocol but after a brief IR exposure to set the system in its PPC state. The latter $\mathrm{G}\left(\mathrm{V}_{\mathrm{g}}\right)$ reveals a more prominent MD than observed in the dark-state.

Note that the conductance in this case increased by $60 \%$ while the magnitude of the memory-dip increased by $150 \%$, the absolute value of the MD component $\delta \mathrm{G}_{\mathrm{MD}}$ increases by more than the average G. This photo-enhanced memory-dip has been observed in all our $\mathrm{GeSb}_{\mathrm{x}} \mathrm{Te}_{\mathrm{y}}$ samples with $R_{\square}>10^{5} \Omega$, and independent of the equilibrium value of $\mathrm{V}_{\mathrm{g}}$ in the dark-state within the range of $-8 \mathrm{~V}$ to $+8 \mathrm{~V}$. It was never encountered in previously studied electron-glasses where, if anything, IR-exposure caused a diminishment of the MD magnitude [36, 40]. Actually in every instance where $\mathrm{G}$ increased, be it due to temperature, non-Ohmic field, or decrease of disorder $\delta \mathrm{G}_{\mathrm{MD}} / \mathrm{G}$ always went down.

Another deviation from the electron-glass norm is observed in the asymptotic dependence of the MD amplitude on time. In previously studied electron-glasses the MD amplitude was found to grow logarithmically with the time the system relaxes under a fixed $V_{\mathrm{g}}$ [35]. In the

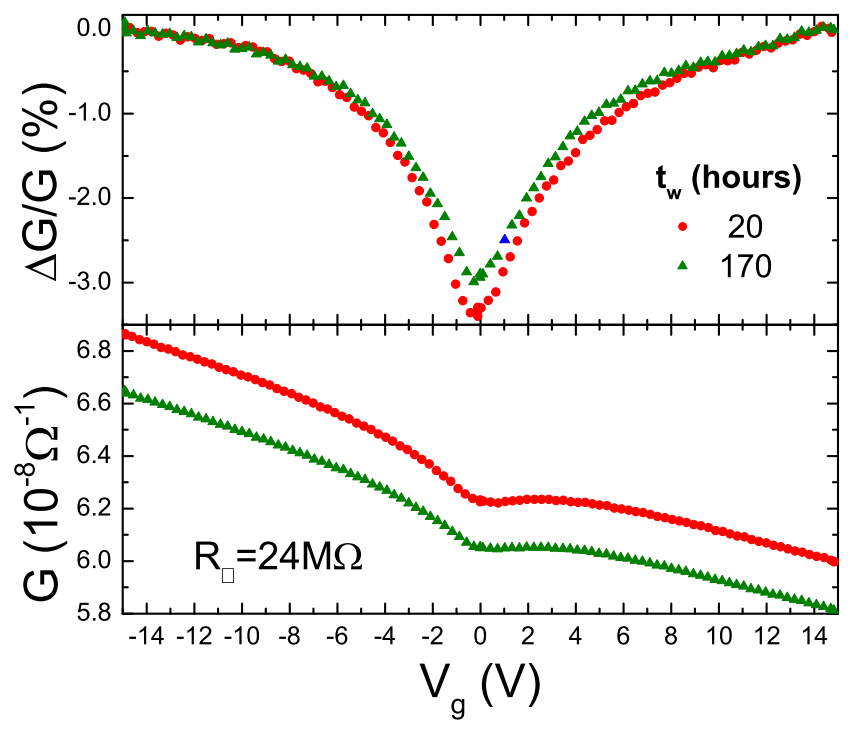

FIG. 10: The asymptotic behavior of the field-effect versus the time elapsed since put in the PPC-state. Bottom graph shows the full $G\left(V_{g}\right)$ curves. Upper graph illustrates that the magnitude of the memory-dip diminishes at long times. The curves in the upper graph were obtained form the $G\left(V_{g}\right)$ data in the bottom graph by subtracting the linear part of the field-effect).

PPC state however, the MD amplitude actually decreases at long times. The example shown in Fig.10 illustrates how, concomitant with the slow decay of the PPC, the MD magnitude diminishes with time.

These observations lead us to consider the following scenario to account for this seemingly paradoxical IR effect.

Let us assume that the sample is not uniform; some regions in it are not part of the electron-glass phase. These could be of two types; regions where the disorder is weak such that locally they are diffusive, and regions in the sample where the carrier-concentration is low. Neither type of a region would exhibit long-lasting electronglass dynamics if probed by itself; conspicuous electronglass effects in field-effect experiments are only detectable in systems where both, high carrier-concentration and strong disorder are present [26]. Therefore, regions that do not meet this requirement would exhibit just a linear $\mathrm{G}\left(\mathrm{V}_{\mathrm{g}}\right)$, reflecting the material thermodynamic DOS dependence on energy, but no memory-dip. On the other hand, they are just as good as other regions to contribute to PPC. Actually, all other things being equal, regions with low carrier-concentration are more likely to be significantly affected by the optical excitation. Once primed by the IR, some of these regions will just exhibit smaller resistance. Some others, having sufficiently large resistance may have their carrier-concentration elevated enough to exhibit memory-dip thus adding weight to the global component. Either way, a larger magnitude of the MD should result in the PPC-state. 


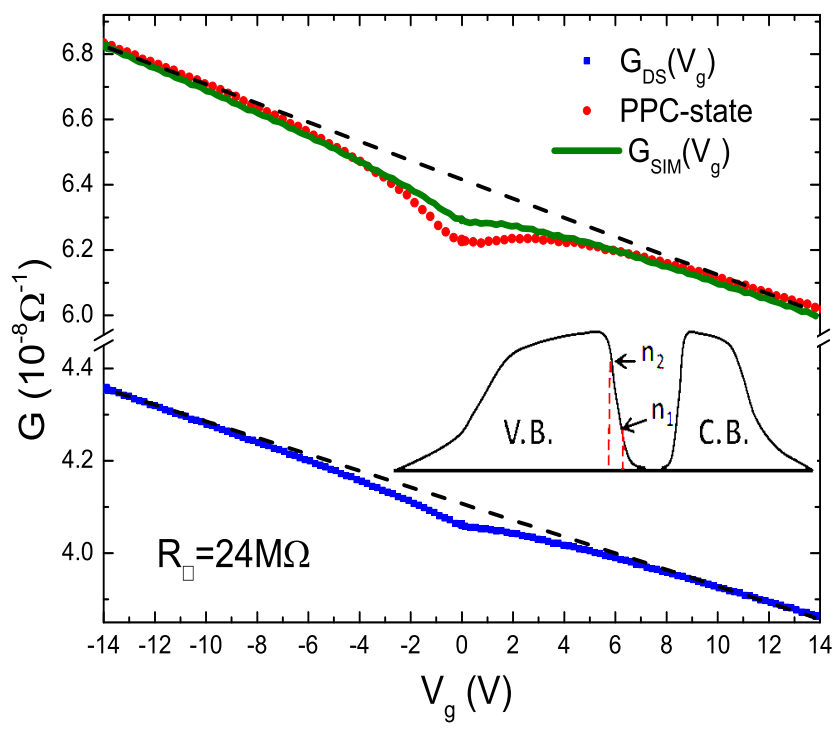

FIG. 11: The field-effect for the $24 \mathrm{M} \Omega$ sample in its darkstate (squares) and in the PPC-state (circles). Dashed lines are the thermodynamic component of $G\left(V_{g}\right)$. The full curve labeled $\operatorname{GSIM}_{\mathrm{SIM}}\left(\mathrm{V}_{\mathrm{g}}\right)$, is the simulated curve (see text for details). This particular sample was chosen for this test due to its large MD amplitude in the dark-state but the same qualitative outcome was observed with other samples. The schematic overlay depicts a "smoothed" density of states for the valence V.B. and conduction band V.B. respectively (following the band-structure calculation of reference [38]). This illustrates why the thermodynamic component part of the field-effect is sloped as in our experiments for both, the state with carrierconcentration $\mathrm{n}_{1}$ as in $\mathrm{n}_{2}$.

A simple check on whether this scenario might be in the right direction to account for the IR-enhancement effect is illustrated in Fig.11. The resistance of the alleged non-glass regions is taken as in series with the electron-glass part of the sample. The field-effect that results in the PPC state, $\mathrm{G}_{\mathrm{SIM}}\left(\mathrm{V}_{\mathrm{g}}\right)$ is then estimated by: $\mathrm{G}_{\mathrm{SIM}}^{-1}\left(\mathrm{~V}_{\mathrm{g}}\right)=\mathrm{G}_{\mathrm{DS}}^{-1}\left(\mathrm{~V}_{\mathrm{g}}\right)-\mathrm{G}_{\mathrm{PAR}}^{-1}\left(\mathrm{~V}_{\mathrm{g}}\right)$ where $\mathrm{G}_{\mathrm{DS}}\left(\mathrm{V}_{\mathrm{g}}\right)$ is the experimentally measured field-effect in the dark-state, and $\delta \mathrm{G}_{\mathrm{PAR}}^{-1}\left(\mathrm{~V}_{\mathrm{g}}\right)$ is the resistance change of the non-glass regions when their dark carrier-concentration $\mathrm{n}_{1}$ is increased to $\mathrm{n}_{2}$ by the IR exposure (see schematic description in Fig.11). The model uses 2 parameters; the slope and the value of $\mathrm{G}_{\mathrm{PAR}}\left(\mathrm{V}_{\mathrm{g}}\right)$ at $\mathrm{V}_{\mathrm{g}}=0$. These are varied to match the overall slope of the experimental $G\left(V_{g}\right)$ in the PPC state. This simple modeling already accounts for a large part of the MD-enhanced effect. To account for the full enhancement it may be necessary to include the contribution from regions that are turned into the electron-glass state when primed by the IR, which will also involve a more refined treatment, probably invoking a percolation model of the inhomogeneous scenario than the simple procedure used above.

There is hardly a need to justify the assumption of inhomogeneity of these samples, being disordered is tan- tamount to being inhomogeneous. The question is how the inhomogeneity manifests itself; what physical property varies in space and above what spatial scale can the system be considered homogeneous? In the present case it is of interest to assess the contribution of fluctuations in carrier-concentration to the quenched disorder.

Spatial variations of carrier-concentration is not uncommon in multi-component systems. It has been recognized for example, as the reason for magneto-transport anomalies in $\mathrm{Hg}_{1-\mathrm{x}} \mathrm{Cd}_{\mathrm{x}} \mathrm{Te}$ films [41] (also a chalcogenide compound). Palm et al referred to the nature of inhomogeneities in their $\mathrm{Hg}_{1-\mathrm{x}} \mathrm{Cd}_{\mathrm{x}} \mathrm{Te}$ samples by: "...different amounts of the insulator's fixed charge in various regions..." stating that ..."this is like having different devices within one sample..." [41].

This type of inhomogeneity appears also in amorphous compounds such as indium-oxide. As pointed out by Givan and Ovadyahu [42], the energy cost of forming charge-gradients in a conducting matter may be mitigated by variation in chemical composition. Inhomogeneous carrier-concentration is especially important when a many-body phenomenon like superconductivity is involved [42]. The sensitivity to carrier-concentration [26, 38] makes it also important for the electron-glass phenomenon.

GeTe-based compounds are as susceptible to this kind of disorder as any other multi-component system. Using heat-treatment, Bahl and Chopra were able to vary the carrier-concentration in their GeTe samples by more than an order of magnitude [43] while preserving the same crystalline symmetry of the material. Crystal chemistry constraints in these materials are therefore not very effective in suppressing stoichiometry fluctuations. This should be compared with the mere factor of 2 variation in carrier-concentration possible in (self-doped) crystalline indium-oxide $\operatorname{In}_{2} \mathrm{O}_{3-\mathrm{x}}$ without doping by foreign elements [40]. Adding a third element (Sb) to the GeTe system apparently only allows for greater variability; reported values for $\mathrm{n}$ in $\mathrm{GeSb}_{\mathrm{x}} \mathrm{Te}_{\mathrm{y}}$ with various $\mathrm{x}$ and $\mathrm{y}$ range between $\approx 3 \cdot 10^{18} \mathrm{~cm}^{-3}\left[32\right.$ to $\approx 10^{22} \mathrm{~cm}^{-3}[29]$. Also, the large dielectric constant of $\mathrm{GeSb}_{\mathrm{x}} \mathrm{Te}_{\mathrm{y}}$ [28] further reduces the energy-cost associated with carrier-concentrations gradients.

To examine the spatial extent of these inhomogeneities, we initiated a study of the variation in the localstoichiometry in $\mathrm{GeSb}_{\mathrm{x}} \mathrm{Te}_{\mathrm{y}}$ films. Recall that these materials are conducting by virtue of vacancies [39] and therefore we expect that mapping stoichiometry may be indicative of local carrier-concentration. This was done by employing energy-dispersive-spectroscopy (EDS) in the STEM mode at $200 \mathrm{kV}$ beam. The methodology is based on building the distribution of the local stoichiometry by performing consecutive EDS reading over a square with lateral side $\mathrm{L}$. The study revealed $\approx 20 \%$ variations in both Ge:Te and Sb:Te atomic-ratios in a distribution based on stoichiometry readings of squares with 
$\mathrm{L}=400 \mathrm{~nm}$ taken across a given sample. A spatial scale of $400 \mathrm{~nm}$ is much larger than typical values of the hoppinglength in strongly-localized samples at $\simeq 4 \mathrm{~K}[44]$. This makes the inhomogeneous scenario plausible; the spatial scale associated with the compositional disorder is large enough to justify treating the sample as being composed of segments with different transport characteristics.

It is natural to ask why this IR-enhancement effect has not been observed, for example, in amorphous indiumoxide where compositional disorder similar to that of $\mathrm{GeSb}_{\mathrm{x}} \mathrm{Te}_{\mathrm{y}}$ has been found [42]. Actually, at least to some degree the effect might be expected to exist in any electron-glass: These systems are inherently inhomogeneous and the notion of regions in the current-carrying network being in series with bottleneck resistances is a natural part of percolation models [45 48] that purport to describe their transport properties.

A likely reason for the absence of this effect in the previously studied electron-glasses is simple: they do not exhibit PPC. To show persistent-photoconductivity there are rather restrictive conditions to be met. The first is feasibility of charge-generation from the lightsource. The optical gap of $\mathrm{GeSb}_{\mathrm{x}} \mathrm{Te}_{\mathrm{y}}$ is $0.4-0.8 \mathrm{eV}$ [27] allowing inter-band transitions which evidently involve generation of excess charge in the system (note however that effective PPC may be achieved sometimes even for smaller photon energies than necessary for interband transitions [49]). Indium-oxide and other previously studied electron-glasses, tested with the same IRprotocol as in this work, do not meet this requirement. The photon energy of the IR source used in these experiments $(\approx 1 \mathrm{eV})$ is smaller than their optical gap [30, 50]. This allows only intra-band transitions, which may be effective in randomizing site-occupation which (under sufficiently large energy flux), diminish the MD [36, 40]. However, no extra charge is generated in the system under these conditions.

A long-lasting PPC state is not guaranteed even when using a source with a photon energy that exceeds the optical gap. To keep the photo-generated electron-hole pairs from recombining and preserve the excess conductance, a mechanism has to exist to create an effective barrier against fast recombination. In general it is hard to identify this mechanism in a given system [7]. It has not yet been identified in $\mathrm{GeSb}_{\mathrm{x}} \mathrm{Te}_{\mathrm{y}}$ either. It would be interesting to extend the study of infrared excitation to GeTe samples, eliminating the $\mathrm{Sb}$, which may help in identifying the defect responsible for in the persistentphotoconductivity we observe in the GeSbTe compounds.

As already mentioned, PPC has been observed in many systems but, to our knowledge, not in a system with carrier-concentration as high as in these $\mathrm{GeSb}_{\mathrm{x}} \mathrm{Te}_{\mathrm{y}}$ films. Enhancing the conductance of a system with $\mathrm{n} \approx 10^{20}$ $10^{21} \mathrm{~cm}^{-3}$ by $10-100 \%$ (Fig. 6 ) seems to require a photoinduced $\delta$ n of not smaller smaller than $\approx 10^{19}-10^{20} \mathrm{~cm}^{-3}$. This may be estimated by comparison to field-effect data; $\delta \mathrm{G}\left(\mathrm{V}_{\mathrm{g}}\right)$ associated with the thermodynamic component results from adding (removing) a charge of $\delta \mathrm{n}$ to (from) the sample. This charge is determined by $\delta \mathrm{V}_{\mathrm{g}}$ and the sample-gate capacitance C. For $\delta \mathrm{V}_{\mathrm{g}}=20 \mathrm{~V}$, and $\mathrm{C} \approx 10 \mathrm{nF} \cdot \mathrm{cm}^{-2}$, the extra charge $\delta \mathrm{n}$ typically associated with the range of our $\mathrm{G}\left(\mathrm{V}_{\mathrm{g}}\right)$ measurements is of order $10^{18}-10^{19} \mathrm{~cm}^{-3}$ (depending on whether the charge is confined to a screening-layer or is spread evenly along the film thickness). The conductance change $\delta \mathrm{G}$ associated with this $\delta$ n varies between $0.7 \%$ to $9.5 \%$ for samples with $\mathrm{R}_{\square}=2.5 \mathrm{k} \Omega$ and $\mathrm{R}_{\square}=24 \mathrm{M} \Omega$ respectively. The conductance increment in the PPC state $\delta \mathrm{G}_{\mathrm{IR}}$ is an order of magnitude larger (see Fig.6), which may suggest that the associated $\delta \mathrm{n}$ is also larger. Perhaps much larger.

To put things is perspective, it is interesting to compare our PPC results with those obtained for another chalcogenide that is well known to exhibit persistentphotoconductivity, $\mathrm{Pb}_{1-\mathrm{x}} \mathrm{Sn}_{\mathrm{x}} \mathrm{Te}$. This compound has similar material characteristics as $\mathrm{GeSb}_{\mathrm{x}} \mathrm{Te}_{\mathrm{y}}$ (chemistry, optical-gap, large dielectric-constant, p-type conductivity). Persistent-photoconductivity in $\mathrm{Pb}_{1-\mathrm{x}} \mathrm{Sn}_{\mathrm{x}} \mathrm{Te}$ compounds doped with In or Ga has been extensively studied 7]. Working with bulk crystals allowed the researchers an effective way to estimate the photoinduced excess charge $\delta \mathrm{n}$ by analyzing Shubnikov-de Haas oscillations data. Values for $\delta \mathrm{n}$, under energy-flux levels of similar magnitude as used in this work, reached only $10^{17}$. $10^{18} \mathrm{~cm}^{-3}$ 7]. Such values appear to be too low to account for our PPC results. This may indicate that photogeneration of charge is more efficient in $\mathrm{GeSb}_{\mathrm{x}} \mathrm{Te}_{\mathrm{y}}$ or that the two processes of inserting the same $\delta$ n to the system affect its conductance differently, perhaps due to the inhomogeneity (an inherent property of the hopping systems). These issues must await a direct measurement of the photoinduced excess-charge in $\mathrm{GeSb}_{\mathrm{x}} \mathrm{Te}_{\mathrm{y}}$ films.

In sum, we presented in this paper experimental results that demonstrate the existence of persistentphotoconductivity in the degenerate semiconductor $\mathrm{GeSb}_{\mathrm{x}} \mathrm{Te}_{\mathrm{y}}$. This non-stoichiometric compound has rather high carrier-concentration and thus it also exhibits electron-glass effects when in the strongly localized regime. It is demonstrated that persistentphotoconductivity and electron-glass phase are different nonequilibrium phenomena. They manifestly may coexist in the strongly localized regime of $\mathrm{GeSb}_{\mathrm{x}} \mathrm{Te}_{\mathrm{y}}$ although it is not entirely clear that they share the same spatial regions in the disordered system. The memory-dip, a distinguishing feature of the electron-glass, is appreciably enhanced In the PPC-state. There is also an intriguing interplay between these phenomena in terms of a dramatic slowdown of the electron-glass dynamics while in the PPC-state that will be treated in future work.

Further work is needed to study the detailed temperature dependence of the persistent-photoconductivity in these materials and to elucidate the nature of the defects that are associated with this effect. 
Illuminating discussions with Dmitry Khokhlov on persistent-photoconductivity are gratefully acknowledged. This research has been supported by a grant administered by the Israel Academy for Sciences and $\mathrm{Hu}-$ manities.

[1] M. G. Craford, G. E. Stillman, J.A. Rossi, and N. Holonyak, Phys. Rev., 168, 867 (1968).

[2] D. V. Lang and R. A. Logan, Phys. Rev. Lett., 39, 635 (1977); D. V. Lang, R. A. Logan, and M. Jaros, Phys. Rev. B 19, 1015 (1979).

[3] H. J. Queisser and D. E. Theodorou, Phys. Rev. Lett., 43, 401 (1979); Jennifer Misuraca, Jelena Trbovic, Jun Lu, Jianhua Zhao, Yuzo Ohno, Hideo Ohno, Peng Xiong, and Stephan von Molnár, Phys. Rev. B 82, 125202, (2010).

[4] P. M. Mooney, Journal of Applied Physics 67, R1 (1990).

[5] J. Y. Lin, A. Dissanayake, G. Brown, and H. X. Jiang, Phys. Rev. B 42, 5855 (1990).

[6] Z. Su and J. W. Farmer, Appl. Phys. Lett. 59, 30 (1991).

[7] B. A. Akimova,. V. Dmitriev., R. Khokhlov, L. I. Ryabova, phys. stat. sol. (a) 137, 9 (1993).

[8] S. Ghosh and V. Kumar, Europhys. Lett., 24 (9), 779 (1993).

[9] T. Y. Lin, H. M. Chen, M. S. Tsai, and Y. F. Chen, F. F. Fang, C. F. Lin and G. C. Chi, PRB 58, 13793 (1998).

[10] D. Jia, J. Zhu, and B. Wu, Journal of The Electrochemical Society, 147 (1) 386 (2000).

[11] V. V. Ursaki, I. M. Tiginyanu, P. C. Ricci, A. Anedda, S. Hubbard, and D. Pavlidis, J. of App. Phys. 94, 3875 (2003).

[12] X. Li, F. Zhong, K. Qiu, Y. Wang, J. Wang, and Y. Wang, AIP Conference Proceedings 893, 291 (2007).

[13] J. H. Davies, P. A. Lee, and T. M. Rice, Phys. Rev. Letters, 49, 758 (1982).

[14] M. Grünewald, B. Pohlman, L. Schweitzer, and D. Würtz, J. Phys. C, 15, L1153 (1982).

[15] M. Pollak and M. Ortuño, Sol. Energy Mater., 8, 81 (1982); M. Pollak, Phil. Mag. B 50, 265 (1984).

[16] G. Vignale, Phys. Rev. B 36, 8192 (1987).

[17] C. C. Yu, Phys. Rev. Lett., 82, 4074 (1999).

[18] M. Müller and L. B. Ioffe, Phys. Rev. Lett. 93, 256403 (2004).

[19] Vikas Malik and Deepak Kumar, Phys. Rev. B 69, 153103 (2004).

[20] R. Grempel, Europhys. Lett., 66, 854 (2004).

[21] Eran Lebanon, and Markus Müller, Phys. Rev. B 72, 174202 (2005).

[22] Ariel Amir, Yuval Oreg, and Yoseph Imry, Annu. Rev. Condens. Matter Phys. 2, 235 (2011).

[23] M. Pollak, M. Ortuño and A. Frydman, "The Electron Glass", Cambridge University Press, England (2013).

[24] Ben Chorin, Z. Ovadyahu and M. Pollak, Phys. Rev. B48, 15025 (1993).
[25] Z. Ovadyahu, Phys. Rev. Lett., 99, 226603 (2007).

[26] Z. Ovadyahu, Phys. Rev. B 88, 085106 (2013); C. R. Physique 14, 700 (2013).

[27] Bong-Sub Lee, J. R. Abelson, S. G. Bishop, Dae-Hwan Kang, Byung-ki Cheong, and Ki-Bum Kim, J. of Appl. Phys., 97, 093509 (2005); Ming Xu, Shen-Jin Wei, Song $\mathrm{Wu}$, Fei Pei, Jing Li, Song-You Wangy and Liang-Yao Chen, J. of the Korean Physical Society, 53, 2265 (2008).

[28] T. Siegrist, P. Jost, H. Volker, M. Woda, P. Merkelbach, C. Schlockermann and M.Wuttig, Nature Mater., 10, 202 (2011).

[29] R. Fallica, F. Volpe, M. Longo, C. Wiemer, O. Salicio, and A. Abrutis, Applied Physics Letters 101, 102105 (2012).

[30] O. Cohen and Z. Ovadyahu, Phys. Rev. B 50, 10442 (1994); Z. Ovadyahu, Phys. Rev. B 78, 195120 (2008).

[31] O. Cohen, Z. Ovadyahu, and M. Rokni, Phys. Rev. Lett., 69, 3555 (1992).

[32] E. Prokhorov, G. Trapaga, and J. González-Hernández, J. Appl. Phys. 104, 103712 (2008).

[33] Z. Ovadyahu, Phys. Rev. B 83, 235126 (2011).

[34] A. Vaknin, Z. Ovadyahu, and M. Pollak, Phys. Rev. B 61, 6692 (2000); J. Bergli and Y. M. Galperin, Phys. Rev. B 85214202 (2012); Y. Meroz, Y. Oreg and Y. Imry, EPL, 105, 37010 (2014).

[35] Z. Ovadyahu, X. M. Xiong, and P. W. Adams, Phys. Rev. B 82, 195404 (2010).

[36] Z. Ovadyahu, Phys. Rev. B 90, 054204 (2014).

[37] A. Vaknin, Z. Ovadyahu, and M. Pollak, Phys. Rev. B 65, 134208 (2002).

[38] A. Vaknin, Z. Ovadyhau, and M. Pollak, Phys. Rev. Lett., 81, 669 (1998).

[39] A. H. Edwards A. C. Pineda, P. A. Schultz, M.s G. Martin, A. P. Thompson, and H.P. Hjalmarson, C. J. Umrigar, J. Phys.: C Condens. Matter 17, L329 (2005); ibid Phys. Rev. B 73, 045210 (2006).

[40] V. Orlyanchik, A.Vaknin, Z. Ovadyahu, and M. Pollak, Phys. Stat. Sol., b230, 61 (2002).

[41] E. C. Palm, W. Szott, P. S. Kobiela, W. P. Kirk, R. A. Schiebel and M. A. Reed, J. Vac. Sci. Technol. A 6 (4), 2716 (1988).

[42] U. Givan and Z. Ovadyahu, Phys. Rev. B 86, 165101 (2012).

[43] S. K. Bahl and K. L. Chopra, Journal of Applied Physics 41, 2196 (1970).

[44] Typical values for hopping length at $\mathrm{T} \approx 4 \mathrm{~K}$ for a variable range hopping system is rarely larger than $\approx 50 \mathrm{~nm}$.

[45] B. I. Shklovskii, A.L. Efros, Sov. Phys. JETP 33, 468 (1971).

[46] V. Ambegaokar, B. I. Halperin, J. S. Langer, Phys. Rev. B 4, 2612 (1971).

[47] M. Pollak, J. Non-Cryst. Solids 11, 1 (1972).

[48] C. H. Seager and G. E. Pike, Phys. Rev. B 10, 1435 (1974).

[49] Shingo Katsumoto, Fumio Komori, Naokatsu Sano, Shun-ichi Kobayashi, J. Phys. Soc. Jpn. 56, 2259, (1987).

[50] Z. Ovadyahu, Phys. Rev B 47, 6161 (1993). 